\title{
PEG-Coated Titania Nanatubes for Controlled Drug Release
}

\author{
Fei Luo \\ College of Chemistry And Chemical Engineering \\ Fujian Normal University \\ Fuzhou, China \\ e-mail: 781069499@qq.com
}

\author{
Guojie Xu \\ College of Chemistry And Chemical Engineering \\ Fujian Normal University \\ Fuzhou, China \\ e-mail: xuguojie-0935@163.com
}

\author{
Yan Liu \\ College of Chemistry And Chemical Engineering \\ Fujian Normal University \\ Fuzhou, China \\ e-mail: 522975901@qq.com
}

\author{
Chunling Xie \\ College of Chemistry And Chemical Engineering \\ Fujian Normal University \\ Fuzhou, China \\ e-mail:xc1197@163.com \\ Xiufeng Xiao* \\ College of Chemistry And Chemical Engineering \\ Fujian Normal University \\ Fuzhou, China \\ e-mail: xfxiao@fjnu.edu.cn
}

\begin{abstract}
Developing coatings on implant surface as drug carriers can reduce organ toxicity and effectively deliver drug locally to the target compared with the oral approach. Titanium dioxide $\left(\mathrm{TiO}_{2}\right)$ nanotube has great potential for this application for widely used Ti implants because of its high surface area, ability to promote bone growth, and biocompatibility. However,there are two issues needed to be solved before further advancing $\mathrm{TiO}_{2}$ nanotubes technology as drug carriers: uncontrolled drug release and poor mechanical properties. PEG by hydrothermal method will load onto titania nanotube arrays coating over the drugloaded titania nanotube arrays to improve the properties of drug release. The samples were characterized by scanning electronic microscope (SEM). The results show that TNTs modified by PEG-coated possess improved drug release characteristics with reduced burst release (from $89 \%$ to $69 \%$ ) and prolonged drug release (from 9 days to over 15 days). This approach provides an alternative to tailor the surface of TNTs and offer considerable prospects for diverse biomedical applications.
\end{abstract}

Keywords- Titania nanotube; PEG; hydrothermal method; ibuprofen; Drug Release

\section{INTRODUCTION}

Titanium (Ti) implant is the most widely used implant material. The dissolution of $\mathrm{Ti}$ into body is very insignificant. The Ti metal surface can spontaneously form a stable and inert layer of titanium dioxide $\left(\mathrm{TiO}_{2}\right.$, titania), which will prevent Ti metal from reacting with body fluid. Delivery of drugs locally from a Ti implant surface has become a commonly used option to prevent infection, reduce organ toxicity, and minimize drug resistance. The benefit of sustained drug release will not only significantly reduce the undesired side effects and the frequency of administering drugs, it may also eliminate the need for self-administration and improve the patient compliance. The $\mathrm{TiO}_{2}$ nanotubes are one of the most attractive nanomaterials for this application because of their biocompatibility, high surface area, ability to promote direct tissue, and bone growth. ${ }^{[1.2]}$ Several methods such as sol-gel, hydrothermal, vapor deposition, and anodization have been used in the synthesis of nanostructured $\mathrm{TiO}_{2}$, from nanopores to nanowires and nanotubes. Since Zwiling et $\mathrm{al}^{[3]}$ and Gong et $\mathrm{al}^{[4]}$ first reported the electrochemical synthesis of synthesized nanoporous and nanotubular $\mathrm{TiO}_{2}$. The science and technology surrounding the growth of TNTs has grown enormously. The nanotubular structures of $\mathrm{TiO}_{2}$ with open volume indicate themselves as one of the ideal candidates as carriers for drug loading and release. 10 However, the issue with $\mathrm{TiO}_{2}$ nanotubes as drug carriers is the short drug elusion time of only several hours in most of the reports. ${ }^{[5,6]}$ For example, Xiao et al. ${ }^{[6]}$ found that $90 \%$ of the total loaded cisplatin released from the $\mathrm{TiO}_{2}$ nanotubes in the first $100 \mathrm{~min}$, no matter how much drug was loaded. Song et al. ${ }^{[5]}$ found that $90 \%$ of horseradish peroxidase was released in the first minute from untreated $\mathrm{TiO}_{2}$ nanotubes and adding hydrophobic monolayer of octadecylphosphonic acid only improved the release rate by less than two orders. TNTs have been fabricated on a range of non-native substrates including silicon, glass, transparent conducting oxide (TCO)-coated glass, polyimide, and stainless steel ${ }^{[7-13]}$. Highly ordered titania nanotube (TNT) arrays on Ti surface fabricated by self-ordering electrochemical anodization have attracted increasing attention due to their excellent biocompatibility, high surface-to-volume ratio, 
controllable dimensions and osseointegration properties [14]. These advantages make TNTs ideal platforms for local drug delivery system ${ }^{[8]}$. When attached to the surfaces of nanotube, PEG has been shown to increase their biocompatibility. ${ }^{[15,16]}$ In this work, ibuprofen was selected as a model drug. Ibuprofen is a nonsteroidal antiinflammatory drug which can improve the resistance to infection and treatment of various moderate pain and inflammation. Clinically, it is mainly used for treating rheumatoid arthritis and osteoarthritis. And we present a dramaticlly easy method to load the PEG onto the $\mathrm{TiO}_{2}$ nanotube arrays by hydrothermal method containing a procedure that the $\mathrm{TiO}_{2}$ nanotube were mixed with the $1 \%$ concentration PEG solution, Solution was placed in a reaction vessel for $3 \mathrm{~h}$ at $85^{\circ} \mathrm{C}$. The process is based on the deposition of a PEG-coated to cover the opening pores of the drug-loaded TNTs with reduced burst release and prolonged release kinetics. The TNTs were loaded with ibuprofen sodium as a model of hydrophilic drug to prove the feasibility of this kind of drug delivery system.

\section{EXPERIMENT SECTION}

\section{A. Fabrication of $\mathrm{TiO}_{2}$ nanotubes arrays}

$\mathrm{Ti}$ sheets were mechanically polished and ultrasonically cleaned in acetone for $15 \mathrm{~min}$, followed by chemically etched in $\mathrm{HNO}_{3} / \mathrm{HF}$ and cleaned with distilled water. Anodized $\mathrm{TiO}_{2}$ nanotube films were synthesized by a two-electrode DC anodization system. A Ti foil acted as the working anode, and a graphite rod was used as the cathode. The two electrodes were separated by a distance of approximately $2 \mathrm{~cm}$. Anode and cathode were put into a beaker containing the desired electrolyte, and the whole system was magnetically stirred. The electrochemical process was performed at a constant voltage of $60 \mathrm{~V}$ for $24 \mathrm{~h}$ in $\mathrm{NH}_{4} \mathrm{~F} /$ glycerol electrolyte $(10$ vol $\% \mathrm{H}_{2} \mathrm{O}$ and $0.5 \mathrm{wt} \% \mathrm{NH}_{4} \mathrm{~F}$ ) at a temperature of $30{ }^{\circ} \mathrm{C}$. After anodization, the samples were rinsed with distilled water and dried at room temperature.

\section{B. Chemical synthesis of PEG-coated titanium dioxide nanotubes.}

In order to synthesize PEG functionalized $\mathrm{TiO}_{2}$ nanotubes, the $\mathrm{TiO}_{2}$ nanotubes were mixed with the $1 \%$ concentration PEG 6000 solution put into a reaction vessel, and then placed in an oven, by hydrothermal method for $3 \mathrm{~h}$ at $85^{\circ} \mathrm{C}$, then washed with distilled water and freeze dried.

\section{- C. Characterization.}

Surface morphologies of the samples were observed by field emission scanning electron microscopy(FESEM,JSM-7500F). The amount of released drug was measured by UV-vis spectroscopy (TU1900).

\section{- D. Drug loading.}

A solution of ibuprofen sodium $(40 \mathrm{mg} / \mathrm{ml})$ was prepared in ethanol and pipetted onto the nanotube surface and gently spread to ensure an even coverage. The samples were allowed to dry under vacuum at room temperature for $2 \mathrm{~h}$. The filling step was repeated 5 times until the solution was visible on the surface of the samples. In vitro drug release was tested by immersing the samples in $10 \mathrm{ml} \mathrm{PBS}\left(\mathrm{pH} \mathrm{7.4)}\right.$ at $37^{\circ} \mathrm{C}$.

\section{RESULTS AND DISCUSSION}

\section{A. Structure and Morphology of Titania Nanotube Arrays after PEG-coated.}

The morphology of the prepared TNT before and after hydrothermal method treatment was characterized by SEM and is summarized in Fig. 1(a) (c) and (e). The thickness of the TNT layer was $10 \mu \mathrm{m}$, and the pore diameters were 210nm. Typical images of PEG-coated TNT are presented in Fig. 1(b), From the image it can be clearly observed in the PEG loaded onto the TNTs. Form Fig. 1(c) we can also clearly observed in the PEG loaded into the TNTs.
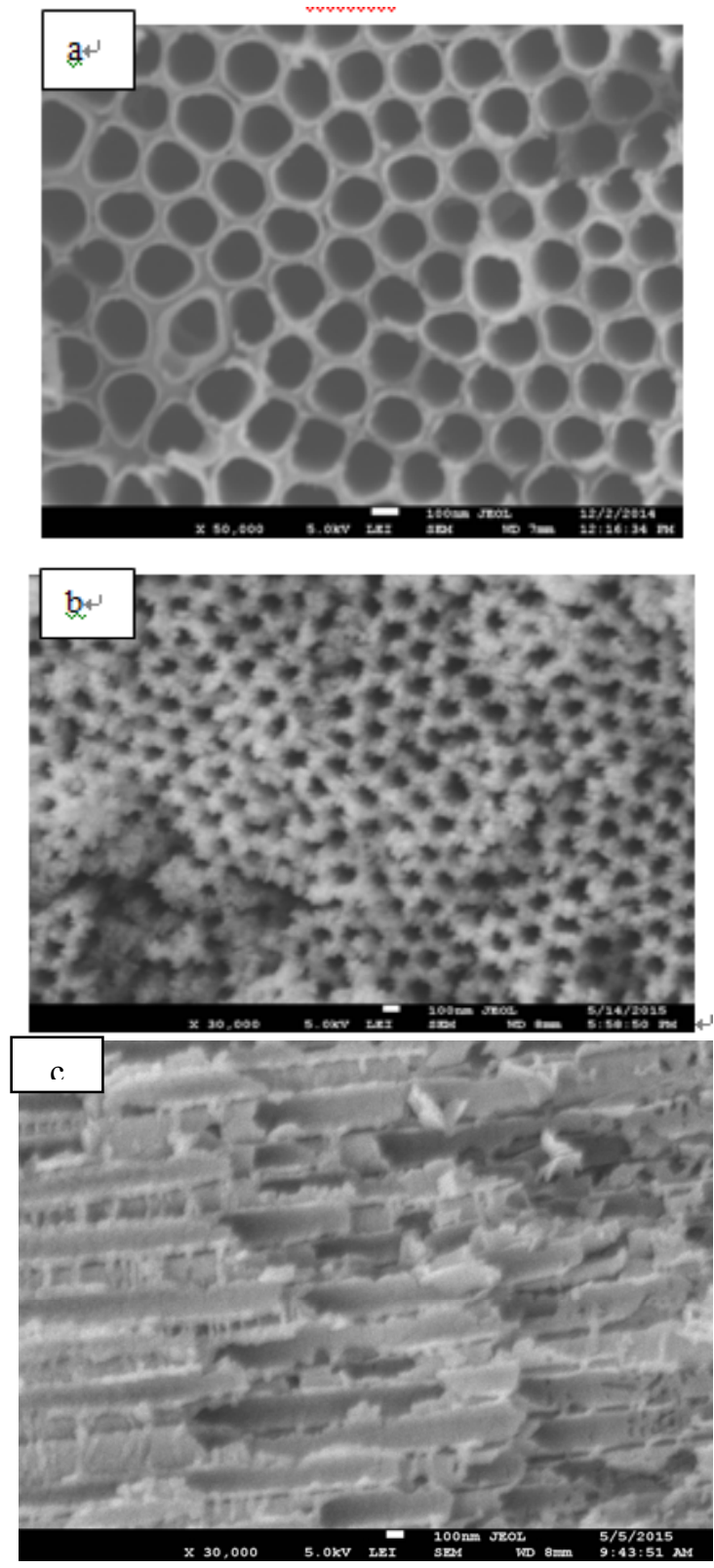

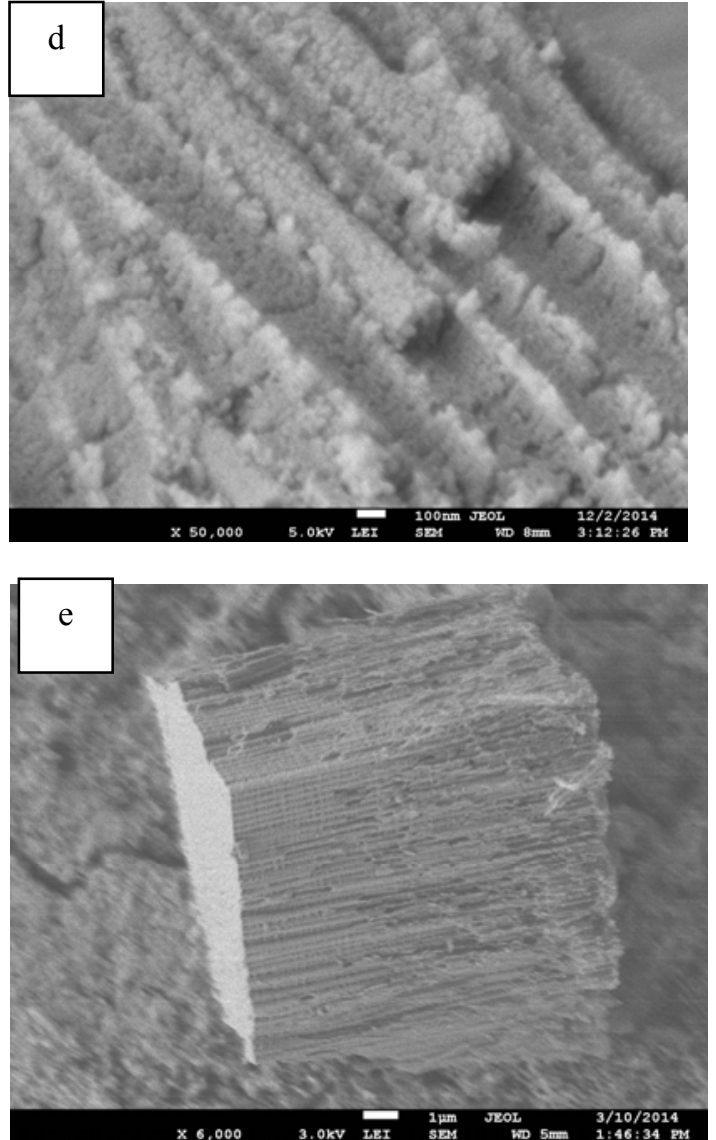

Figure 1. SEM images of $\mathrm{TiO}_{2}$ nanotube arrays fabricated by electrochemical anodizationwithout PEG-coated TNTs:(a) top and (c) /(e)cross section view, and PEGcoated TNTs: (b) top and (d) cross section view.

\section{B. In vitro release of ibuprofen sodium from titania nanotube arrays.}

Comparative drug release curves of ibuprofen sodium loaded into TNTs with and without PEG coating are presented in Fig.2. For uncoated sample, the release kinetics can be described in two stages, with a large amount (89\%) of the drug in the first $12 \mathrm{~h}$ (burst release), and slow release over 12 days. For sample PEG-coated on the surface of TNTs after hydrothermal method, reduced burst release $(69 \%)$ and prolonged drug release (over 15 days) was observed. It was concluded that PEG coating deposited over drug-loaded TNTs by hydrothermal method provides a lower burst and longer release time.

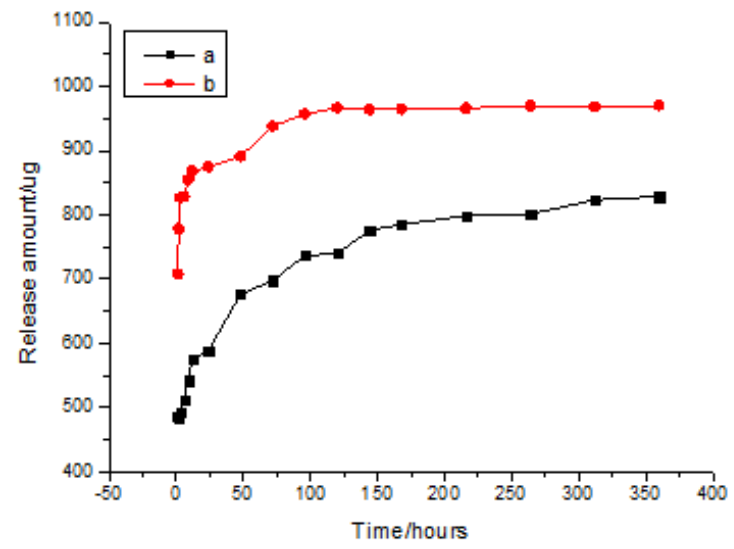

Figure 2. Comparative drug release curves of ibuprofen sodium loaded into TNTs (a)PEG-coated TNTs; (b) TNTs without PEG

\section{CONCLUSION}

The present work shows that PEG-coated was successfully deposited onto titania nanotube arrays by hydrothermal method and significant changes (reduced burst release from $89 \%$ to $69 \%$ and prolonged drug release from 9 days to more than 15 days) in the drug release curve were observed.

\section{ACKNOWLEDGMENT}

This work was financially supported by National Nature Science Foundation of China (30970887), the Science Research Foundation of Ministry of Health (WKJ 2008-2-037), Fujian Province Nature Science Foundation (2011J06019, 2012J01194).

\section{REFERENCES}

[1]. Yao C, Perla V, McKenzie J L, Slamovich E B, Webster T J. Anodized $\mathrm{Ti}$ and $\mathrm{Ti}(6) \mathrm{Al}(4) \mathrm{V}$ possessing nanometer surface features enhances osteoblast adhesion $[\mathrm{J}]$. Biomed Nanotechnol, 2005, 1:68-73.

[2]. Von Wilmowsky C, Bauer S, Lutz R, Meisel M, Neukam F W, Toyoshima T, Schmuki P, Nkenke E, Schlegel K A. In vivo evaluation of anodic $\mathrm{TO}_{2}$ nanotubes: An experimental study in the pig [J]. Biomed Mater Res B Appl Biomater, 2009, 89:165-171.

[3] V. Zwilling, E. Darque-Ceretti, A. Boutry-Forveille, D. David, M. Y. Perrin, and M. Aucouturier, Surf. Interf. Anal. 1999, 27(7): 629637 .

[4] D. Gong, C A Grimes, O K.Varghese, W C. Hu, R. S. Singh, Z. Chen, and E. C. Dickey, [J]. Mater. Res, 2001, 16(12): 33313334 .

[5] Song Y Y, Schmidt-Stein F, Bauer S, Schmuki P. Amphiphilic $\mathrm{TiO}_{2}$ nanotube arrays: An actively controllable drug delivery system. [J] Am Chem Soc, 2009, 131: 4230-4232.

[6] Xiao XL, Yang LX, Guo ML, Pan CF, Cai QY, Yao SZ. Biocompatibility and in vitro antineoplastic drug-loaded trialof titania nanotubes prepared by anodic oxidation of a pure titanium. Sci China, Ser B, 2009, 52: 2161-2165.

[7] G. K. Mor, O. K. Varghese, M. Paulose, and C. A. Grimes, Adv. Funct. Mater, 2005, 15(8), 1291-1296.

[8] J. M. Macak, H. Tsuchiya, S. Berger, S. Bauer, S. Fujimoto, and P. Schmuki, Chem. Phys. Lett, 2006, 428(4-6): 421-425.

[9] H. D. Zheng, A. Z. Sadek, M. Breedon, D. Yao, K. Latham, [J]. du Plessis, and K. Kalantar-Zadeh, Electrochem. Commun, 2009, 11(6): 1308-1311. 
[10] T. Stergiopoulos, A. Valota, V. Likodimos, T. Speliotis, D. Niarchos, P. Skeldon, G. E. Thompson, and P. Falaras, Nanotechnology, 2009, 20(36): 365601.

[11] V. Galstyan, A. Vomiero, E. Comini, G. Faglia, and G. Sberveglieri, RSC Adv, 2011, 1(6): 1038-1044.

[12] S. Farsinezhad, A. Mohammadpour, A. N. Dalrymple, J. Geisinger, P. Kar, M. J. Brett, and K. Shankar, [J]. Nanosci. Nanotechnol, 2013, 13(4): 2885-2891.

[13] J. Tao, T. Wu, and P. Gao, [J]. Nanosci. Nanotechnol, 2012, 12(3): $1852-1858$.
[14] M. S. Aw, J. A. Mensah and D. Losic: ChemComm Vol. 8(2012), p. 3348.

[15] M. S. Aw, K. Gulati and D. Losic: Journal of Biomaterials and Nanobiotechnology Vol. 2(2011), p. 477.

[16]A Sawhney, C Pathak, J Hubbell, Interfacial photopolymerization of poly (ethylene glycol)-based hydrogels upon alginate poly(Llysine) microcapsules for enhanced biocompatibility, Biomaterials, 1993,

14

1008-1016. 